\title{
An Elementary Analysis of the Probability That a Binomial Random Variable Exceeds Its Expectation
}

\author{
Benjamin Doerr \\ Laboratoire d'Informatique (LIX) \\ École Polytechnique \\ Palaiseau \\ France
}

January 8, 2018

\begin{abstract}
We give an elementary proof of the fact that a binomial random variable $X$ with parameters $n$ and $0.29 / n \leq p<1$ with probability at least $1 / 4$ strictly exceeds its expectation. We also show that for $1 / n \leq p<1-1 / n$, $X$ exceeds its expectation by more than one with probability at least 0.0370 . Both probabilities approach $1 / 2$ when $n p$ and $n(1-p)$ tend to infinity.
\end{abstract}

\section{Introduction}

Let $X$ be a random variable following a binomial distribution with parameters $n$ and $p$, that is, we have $\operatorname{Pr}[X=i]=\left(\begin{array}{c}n \\ i\end{array}\right) p^{i}(1-p)^{n-i}$ for all $i \in[0 . . n]$. Then, apart from maybe extreme cases, it seems very natural that with reasonable probability $X$ is at least its expectation $E[X]$ or even exceeds it. Surprisingly, and despite the fact that such statements are very important in the machine learning literature, only very recently rigorous proofs of such statements appeared. We refer to Greenberg and Mohri [GM14] for a detailed discussion on the previous lack of such results.

Prior to the work of Greenberg and Mohri, apart from general bounds like those in Slud [Slu77, apparently only a result of Rigollet and Tong [RT11 was known. This result is stated as $\operatorname{Pr}[X \geq E[X]] \geq \min \left\{p, \frac{1}{4}\right\}$ for all $p \leq \frac{1}{2}$ in the 
paper (Lemma 6.4), but the proof shows the stronger statement

$$
\operatorname{Pr}[X \geq E[X]] \geq \begin{cases}\frac{1}{4} & \text { if } p \in\left[\frac{1}{n}, \frac{1}{2}\right] \\ p & \text { if } p<\frac{1}{n} .\end{cases}
$$

The main work in the proof is showing another interesting result, namely that for all $k \in\left[2 . . \frac{n}{2}\right]$ one has

$$
\operatorname{Pr}\left[\operatorname{Bin}\left(n, \frac{k}{n}\right) \geq k+1\right] \geq \operatorname{Pr}\left[\operatorname{Bin}\left(n, \frac{k-1}{n}\right) \geq k\right] .
$$

The proof of this result uses a connection between binomial distributions and order statistics of uniform distributions (to be found in Section 7.2 of the second volume of Feller [Fel71]) and then proceeds by showing the inequality

$$
k \int_{0}^{\frac{k-1}{n}} t^{k-1}(1-t)^{n-k} d t \leq(n-k) \int_{0}^{\frac{k}{n}} t^{k}(1-t)^{n-k-1} d t .
$$

It is not clear how to extend (11) to $p>\frac{1}{2}$. Note that neither (21) nor this equation with the inequality reversed are true for all $k \in\left[\frac{n}{2} . . n-1\right]$. Hence the following relatively recent result of Greenberg and Mohri appears to be the first one treating the problem in full generality.

Lemma 1 (Greenberg and Mohri GM14]). Let $n \in \mathbb{N}$ and $\frac{1}{n}<p \leq 1$. Let $X \sim \operatorname{Bin}(n, p)$. Then

$$
\operatorname{Pr}[X \geq E[X]]>\frac{1}{4}
$$

This result has found applications not only in machine learning, but also in randomized algorithms, see, e.g., [KKK16, $\mathrm{BCN}^{+} 17$, MM17]. While the result is very simple, the proof is not and uses the Camp-Paulson normal approximation to the binomial cumulative distribution function.

Via a different, again non-elementary proof technique, using among others the hazard rate order and the likelihood ratio order of integer-valued distributions, the following result was shown by Pelekis and Ramon [PR16].

Lemma 2 (Pelekis and Ramon [PR16]). Let $n \in \mathbb{N}$ and $\frac{1}{n} \leq p \leq 1-\frac{1}{n}$. Let $X \sim \operatorname{Bin}(n, p)$. Then

$$
\operatorname{Pr}[X \geq E[X]] \geq \frac{1}{2 \sqrt{2}} \frac{\sqrt{n p(1-p)}}{\sqrt{n p(1-p)+1}+1} .
$$



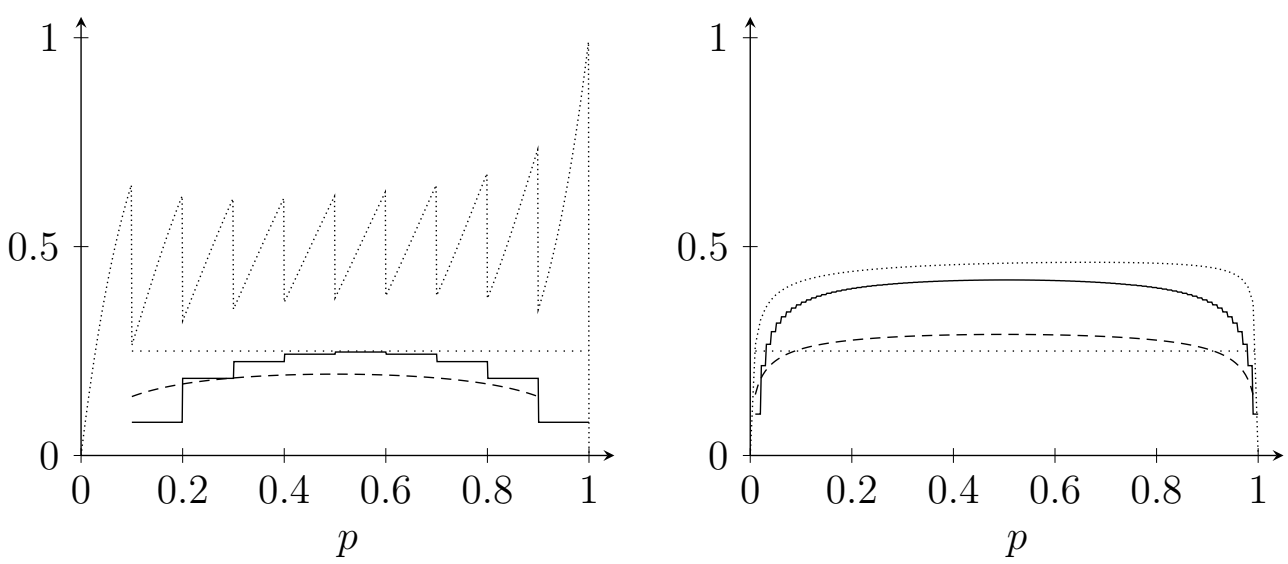

Figure 1: Comparison of the previous bounds of [GM14] (Lemma 1, sparsely dotted lines) and [PR16] (Lemma 2, dashed lines), of our bound (equation (3), solid lines), and of the true value for $\operatorname{Pr}[\operatorname{Bin}(n, p)>n p]$ (dotted lines) for $n=10$ (left) and $n=100$ (right). To increase the readability, for $n=100$ the true value is only depicted at the local minima $\left\{0, \frac{1}{n}, \frac{2}{n}, \ldots, 1\right\}$.

Lemma 2 improves the bound of Lemma 1 when $n p(1-p)>8$, which in particular requires $n>32$ and $E[X]=n p>8$. It however never gives a bound better than $\frac{1}{2 \sqrt{2}} \approx 0.3536$.

In this work, we show that also truly elementary arguments give interesting results for this problem. We prove in Lemma 8 that for $\frac{1}{n} \leq p<1$ and $k:=\lfloor n p\rfloor$, we have

$$
\operatorname{Pr}[X>E[X]]>\frac{1}{2}-\sqrt{\frac{n}{2 \pi k(n-k)}} .
$$

This bound is not perfectly comparable to the previous, but Figure 1 indicates that it is often superior. It has the particular advantage that it tends to $\frac{1}{2}$ when $n p$ and $n(1-p)$ tend to infinity. Our bound does not immediately imply the $\frac{1}{4}$ bound of Greenberg and Mohri GM14, however elementary analyses of a few "small cases" suffice to obtain in Theorem 10 that $\operatorname{Pr}[X>E[X]] \geq \frac{1}{4}$ for all $0.2877 \frac{1}{n} \approx \ln \left(\frac{4}{3}\right) \frac{1}{n} \leq p<1$. The strict version $\operatorname{Pr}[X>E[X]]>\frac{1}{4}$ of the claim is also valid except when $n=2$ and $p=\frac{1}{2}$.

We also show that for $\frac{1}{n} \leq p<1-\frac{1}{n}, X$ exceeds its expectation by more than one with probability at least 0.0370 , again with better bounds available when $n p$ and $n(1-p)$ are larger, see Theorem 12. Such a statement was recently needed in the analysis of an evolutionary algorithm (in the proof of Lemma 3 of the extended version of [DGWY17]). 


\section{Preliminaries}

All notation we shall use is standard and should need not much additional explanation. We denote by $\mathbb{N}:=\{1,2, \ldots\}$ the positive integers. For intervals of integers, we write $[a . . b]:=\{x \in \mathbb{Z} \mid a \leq x \leq b\}$. We use the standard definition $0^{0}:=1\left(\right.$ and not $\left.0^{0}=0\right)$.

It is well-known that $\left(1-\frac{1}{r}\right)^{r}$ is monotonically increasing and that $\left(1-\frac{1}{r}\right)^{r-1}$ is monotonically decreasing in $r$ (and that both converge to $\frac{1}{e}$ ). We need two slightly stronger statements in this work (Lemma 3 (a) and (c)).

Lemma 3. (a) For all $\alpha \geq 0$, the expression $\left(1-\frac{1}{x}\right)^{x-0.5+\alpha}$ is increasing for $x \geq 1$.

(b) For all $\alpha \geq 0$, the expression $\left(1+\frac{1}{x}\right)^{x+0.5+\alpha}$ is decreasing for $x>0$.

(c) The expression $\left(1-\frac{1}{x}\right)^{x}+\left(1-\frac{1}{x}\right)^{x-1}=2\left(1-\frac{1}{2 x}\right)\left(1-\frac{1}{x}\right)^{x-1}$ is decreasing for $x \geq 1$.

Proof. To prove the first part, it suffices to show that $f(x):=\left(1-\frac{1}{x}\right)^{x-0.5}$ is increasing for $x \geq 1$. It is obvious that $0=f(1)<f(x)$ for all $x>1$, so we can concentrate on the case $x>1$. We show that $\ln (f(x))$ is increasing for $x>1$. Using the series expansion of the natural logarithm, we compute $\ln (f(x))=(x-$ $\left.\frac{1}{2}\right) \ln \left(1-\frac{1}{x}\right)=\left(x-\frac{1}{2}\right) \sum_{i=1}^{\infty}\left(-\frac{1}{i x^{i}}\right)=\sum_{i=0}^{\infty}\left(-\frac{1}{(i+1) x^{i}}\right)+\sum_{i=1}^{\infty} \frac{1}{2 i x^{i}}=-1-\sum_{i=1}^{\infty} \frac{i-1}{2 i(i+1)} \frac{1}{x^{i}}$, which is a sum of constant and increasing functions.

The second claim follows from noting that the reciprocal of our expression, $\frac{1}{\left(1+\frac{1}{x}\right)^{x+0.5+\alpha}}=\left(1-\frac{1}{x+1}\right)^{(x+1)-0.5+\alpha}$, is increasing for $x \geq 0$ by the first part.

The third claim follows along similar arguments as the first one. Since $f(x)=$ $2\left(1-\frac{1}{2 x}\right)\left(1-\frac{1}{x}\right)^{x-1}$ is continuous in $x=1$, it suffices to show the claim for $x>1$. For $x>1$, we regard $\ln \left(\frac{1}{2} f(x)\right)$ and compute $\ln \left(\frac{1}{2} f(x)\right)=\ln \left(1-\frac{1}{2 x}\right)+(x-1) \ln \left(1-\frac{1}{x}\right)=$ $-1+\sum_{i=1}^{\infty}\left(\frac{1}{i+1}-\frac{1}{2^{i}}\right) \frac{1}{i} \frac{1}{x^{i}}$, which is a sum of constant and decreasing functions.

From the following version of Stirling's formula we obtain an estimate for binomial coefficients and from that an estimate for $\operatorname{Pr}\left[\operatorname{Bin}\left(n, \frac{k}{n}\right)=k\right]$.

Theorem 4 (Robbins [Rob55]). For all $n \in \mathbb{N}$,

$$
n !=\sqrt{2 \pi n}\left(\frac{n}{e}\right)^{n} R_{n}
$$

where $1<\exp \left(\frac{1}{12 n+1}\right)<R_{n}<\exp \left(\frac{1}{12 n}\right)<1.08690405$.

Corollary 5. For all $n \in \mathbb{N}$ and $k \in[1 . . n-1]$,

$$
\left(\begin{array}{l}
n \\
k
\end{array}\right)=\frac{1}{\sqrt{2 \pi}} \sqrt{\frac{n}{k(n-k)}}\left(\frac{n}{k}\right)^{k}\left(\frac{n}{n-k}\right)^{n-k} R_{n k},
$$




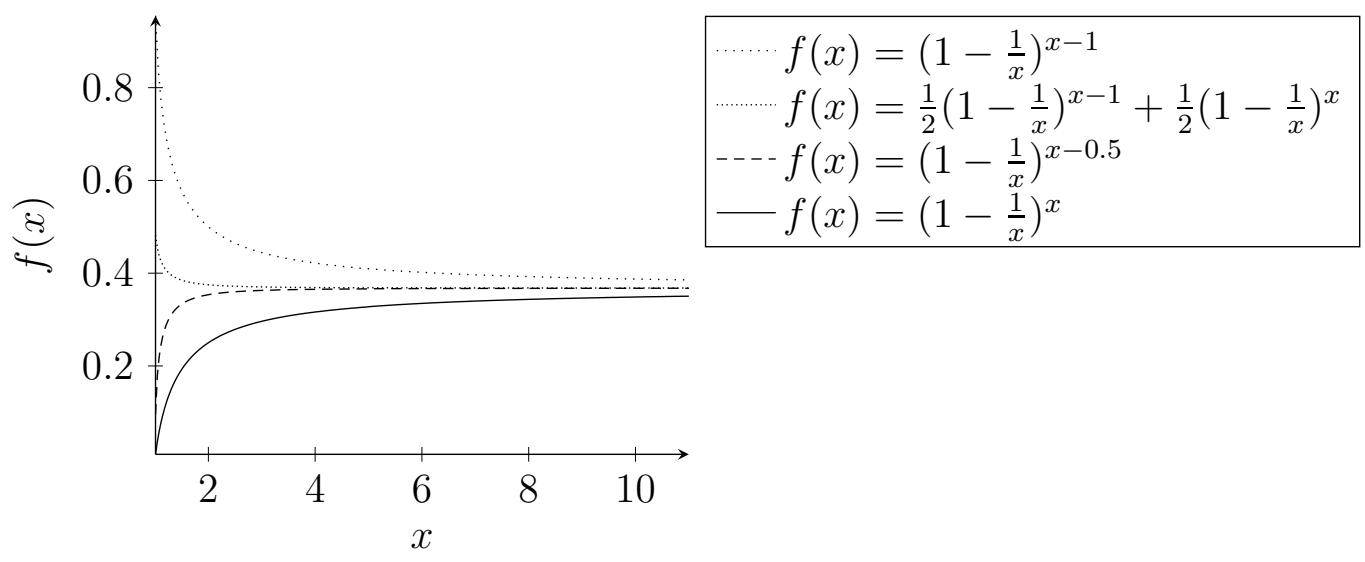

Figure 2: Plots related to Lemma 3 .

where $0.88102729 \ldots=\exp \left(-\frac{1}{6}+\frac{1}{25}\right) \leq \exp \left(-\frac{1}{12 k}-\frac{1}{12(n-k)}+\frac{1}{12 n+1}\right)<R_{n k}<$ $\exp \left(-\frac{1}{12 k+1}-\frac{1}{12(n-k)+1}+\frac{1}{12 n}\right)<1$.

Lemma 6. Let $n \in \mathbb{N}$ and $k \in[1 . . n-1]$. Then $\operatorname{Pr}[X=k]<\frac{1}{\sqrt{2 \pi}} \sqrt{\frac{n}{k(n-k)}}$.

Proof. With Corollary 5 we estimate

$$
\begin{aligned}
\operatorname{Pr}[X=k] & \leq \operatorname{Pr}[Y=k] \\
& =\left(\begin{array}{l}
n \\
k
\end{array}\right)\left(\frac{k}{n}\right)^{k}\left(1-\frac{k}{n}\right)^{n-k} \\
& <\frac{1}{\sqrt{2 \pi}} \sqrt{\frac{n}{k(n-k)}} .
\end{aligned}
$$

A simple coupling argument establishes the natural fact that a binomial distribution with smaller $p$-value is dominated (in a strict sense) by one with larger $p$-value. Very similar results were used and proven also in GM14 (Lemma 1) and [PR16] (Lemma 2.4), however, with more complicated and less intuitive proofs (via differentiating the function $p \mapsto \operatorname{Pr}[\operatorname{Bin}(n, p) \geq n p]$ in [GM14] and via arguing that $\operatorname{Bin}(n, p)$ is smaller than $\operatorname{Bin}(n, q)$ in the likelihood order in [PR16]).

Lemma 7. Let $n \in \mathbb{N}$ and $0 \leq p<q \leq 1$. Let $X \sim \operatorname{Bin}(n, p)$ and $Y \sim \operatorname{Bin}(n, q)$. Then for all $k \in[0 . . n], \operatorname{Pr}[X \geq k]<\operatorname{Pr}[Y \geq k]$.

Proof. Let $R_{1}, \ldots, R_{n}$ be independent random variables uniformly distributed in $[0,1)$. Let

$$
\tilde{X}:=\left|\left\{i \in[1 . . n] \mid R_{i}<p\right\}\right|,
$$




$$
\tilde{Y}:=\left|\left\{i \in[1 . . n] \mid R_{i}<q\right\}\right|
$$

By construction, we have $\tilde{X} \leq \tilde{Y}$ and thus $\operatorname{Pr}[\tilde{X} \geq k] \leq \operatorname{Pr}[\tilde{Y} \geq k]$. Since the event " $\tilde{X}=k-1$ and $\tilde{Y}=k$ " appears with positive probability, we even have $\operatorname{Pr}[\tilde{X} \geq k]<\operatorname{Pr}[\tilde{Y} \geq k]$. Also by construction, $\tilde{X} \sim X$ and $\tilde{Y} \sim Y$, so the previous statement is also valid for $X$ and $Y$.

\section{Proofs of Our Results}

We are now ready to prove our results.

Lemma 8. Let $n \in \mathbb{N}$ and $\frac{1}{n} \leq p<1$. Let $X \sim \operatorname{Bin}(n, p)$. Let $k=\lfloor n p\rfloor$. Then

$$
\operatorname{Pr}[X>E[X]]>\frac{1}{2}-\sqrt{\frac{n}{2 \pi k(n-k)}}=: g(n, k) .
$$

Proof. We compute

$$
\begin{aligned}
\operatorname{Pr}[X>E[X]] & =\operatorname{Pr}[X \geq k+1] \\
& \geq \operatorname{Pr}\left[\operatorname{Bin}\left(n, \frac{k}{n}\right) \geq k+1\right] \\
& =\operatorname{Pr}\left[\operatorname{Bin}\left(n, \frac{k}{n}\right) \geq k\right]-\operatorname{Pr}\left[\operatorname{Bin}\left(n, \frac{k}{n}\right)=k\right] \\
& >\frac{1}{2}-\sqrt{\frac{n}{2 \pi k(n-k)}} .
\end{aligned}
$$

Here the first inequality stems from the natural stochastic domination relation between binomial distributions with different success probabilities (Lemma 7). Last estimate uses (i) the well-known fact that a binomial distribution with integral expectation has this expectation as (unique) median [Neu66] and (ii) the estimate from Lemma 6 .

Note that when $n$ is fixed, then $g(n, k)$ is minimal for $k=1$ and $k=n-1$. Also, $g(n, 1)=g(n, n-1)$ is monotonically increasing in $n$. Hence for all $n \geq 3$, $\frac{1}{n} \leq p<1$, and $k:=\lfloor n p\rfloor$, we have $\operatorname{Pr}[X>E[X]]>g(n, k) \geq g(n, 1) \geq g(3,1)>$ 0.0113 . For $n=2$ and consequently $p \geq \frac{1}{2}$, we compute $\operatorname{Pr}[X>E[X]] \geq \operatorname{Pr}[X=$ $2]=p^{2} \geq \frac{1}{4}$. Hence Lemma 8 immediately gives a constant lower bound for the probability to exceed the expectation. Since we expect that the precise constant of $\frac{1}{4}$ shown below is not important in several applications, e.g., in the runtime analysis of algorithms, we formulate this elementary result explicitly. We add the trivial observation that this result, by possibly lowering the constant, can be extended to smaller values of $p$ as long as they are at least $\frac{\varepsilon}{n}$ for some $\varepsilon>0$. 
Corollary 9. Let $\varepsilon>0$. Then for all $n \in \mathbb{N}, \frac{\varepsilon}{n} \leq p<1$, and $X \sim \operatorname{Bin}(n, p)$, we have $\operatorname{Pr}[X>E[X]]>\min \left\{e^{-\varepsilon}, 0.0113\right\}$.

Proof. For $p \geq \frac{1}{n}$, the claim follows from the above discussion. For $0<p<\frac{1}{n}$, we compute $\operatorname{Pr}[X>E[X]]=1-\operatorname{Pr}[X=0]=1-(1-p)^{n}>1-\exp (-p n)$, using the well-known estimate $1+x<e^{x}$ valid for all $x \neq 0$.

We now show how to improve the lower bound to $\frac{1}{4}$. Since $g(n, k)$ approaches $\frac{1}{2}$ when $k$ and $n-k$ tend to infinity, it is clear that we only have to deal with "small cases". More specifically, since $g(20,3)=g(20,17) \geq 0.2501$, we need to regard for arbitrary $n$ the cases that $p<\frac{3}{n}$ and $p \geq 1-\frac{3}{n}$. In addition, we need to consider the finite number of cases where $n \leq 19$. This will give the following result.

Theorem 10. Let $n \in \mathbb{N}$ and $\frac{\alpha}{n} \leq p<1$, where $\alpha:=\ln \left(\frac{4}{3}\right)<0.2877$. Let $X \sim \operatorname{Bin}(n, p)$. Then $\operatorname{Pr}[X>E[X]] \geq \frac{1}{4}$.

Proof. For $p \in\left[\frac{\alpha}{n}, \frac{1}{n}\right)$ with $\alpha:=\ln \left(\frac{4}{3}\right)<0.2877$, we compute $\operatorname{Pr}[X>E[X]]=$ $\operatorname{Pr}[X \geq 1]=1-(1-p)^{n}>1-\exp (-p n) \geq 1-\exp (-\alpha) \geq \frac{1}{4}$ by choice of $\alpha$.

We assume from now on that $p \geq \frac{1}{n}$. Let $Y \sim \operatorname{Bin}\left(n, \frac{k}{n}\right)$ with $k=\lfloor n p\rfloor$. By Lemma 7.

$$
\operatorname{Pr}[X>E[X]]=\operatorname{Pr}[X \geq k+1] \geq \operatorname{Pr}[Y \geq k+1] .
$$

Hence it suffices to show

$$
\operatorname{Pr}[X \geq k+1] \geq \frac{1}{4} \text { with } X \sim \operatorname{Bin}\left(n, \frac{k}{n}\right)
$$

for all $n \in \mathbb{N}$ and $k \in[1 . . n-1]$. To this aim, let us assume that $X \sim \operatorname{Bin}\left(n, \frac{k}{n}\right)$ in the remainder of this proof. We start by treating the "small" cases $k \in\{1,2, n-$ $3, n-2, n-1\}$.

For $k=1$ and $n \geq 3$, we compute $\operatorname{Pr}[X \geq 2]=1-\operatorname{Pr}[X=0]-\operatorname{Pr}[X=1]=$ $1-\left(1-\frac{1}{n}\right)^{n}-\left(1-\frac{1}{n}\right)^{n-1} \geq \frac{7}{27}>0.2592$, where the first inequality stems from the fact that $n \mapsto\left(1-\frac{1}{n}\right)^{n}+\left(1-\frac{1}{n}\right)^{n-1}$ is decreasing (Lemma 3) and $n \geq 3$.

For $k=2$ and $n \geq 5$, in a similar fashion we compute $\operatorname{Pr}[X \geq 3]=1-\left(1-\frac{2}{n}\right)^{n}-$ $2\left(1-\frac{2}{n}\right)^{n-1}-2\left(1-\frac{1}{n}\right)\left(1-\frac{2}{n}\right)^{n-2}=1-\left(1-\frac{2}{n}\right)^{n-1}\left(5+\frac{4}{(n-2) n}\right)>1-e^{-2}\left(5+\frac{4}{(n-2) n}\right) \geq$ $1-e^{-2}\left(5+\frac{4}{15}\right)>0.2872$ by noting that $\left(1-\frac{2}{n}\right)^{n-1}=\left(\left(1-\frac{1}{n / 2}\right)^{n / 2-1 / 2}\right)^{2}$ is increasing (Lemma 3) and tending to $e^{-2}$.

For $k=n-1$ and $n \geq 2$, we estimate $\operatorname{Pr}[X \geq n]=\left(1-\frac{1}{n}\right)^{n} \geq \frac{1}{4}$ using Lemma 3 and $n \geq 2$.

For $k=n-2$ and $n \geq 4$, we estimate $\operatorname{Pr}[X \geq n-1]=2\left(1-\frac{2}{n}\right)^{n-1}+\left(1-\frac{2}{n}\right)^{n} \geq$ $2\left(\frac{1}{2}\right)^{3}+\left(\frac{1}{2}\right)^{4}=0.3125$ using Lemma 3 and $n \geq 4$.

For $k=n-3$ and $n \geq 6$, we estimate $\operatorname{Pr}[X \geq n-2]=\frac{9}{2}\left(1-\frac{1}{n}\right)\left(1-\frac{3}{n}\right)^{n-2}+$ $3\left(1-\frac{3}{n}\right)^{n-1}+\left(1-\frac{3}{n}\right)^{n}>\frac{9}{2}\left(1-\frac{3}{n}\right)^{n-1}+3\left(1-\frac{3}{n}\right)^{n-1}+\left(1-\frac{3}{n}\right)^{n} \geq 0.25$, using Lemma 3 and $n \geq 6$. 
With this case distinction, we have proven (5) for all $n \in \mathbb{N}$ and $k \in\{1,2, n-$ $3, n-2, n-1\} \cap[1 . . n-1]$. Since $g(20,3)=g(20,17)>0.25$, the concavity of $k \mapsto g(n, k)$ gives $g(20, k)>0.25$ for all $k \in[3 . .17]$. Since for all $n \in \mathbb{N}$ and $k \in[1 . . n-1]$ we have $g(n+1, k)>g(n, k)$ and $g(n+1, k+1)>g(n, k)$, we have $g(n, k)>0.25$ for all $n \geq 20$ and $k \in[3 . . n-3]$, which proves (5) for all $n \geq 20$.

Hence it remains to show (5) for all $n \leq 19$ and $k \in$ [3..n-4]. In principle, these 91 cases can easily be checked in an automated fashion. If we prefer a human-readable proof, we can argue as follows.

For the case $k=3$ and $n \geq 7$, we compute $\operatorname{Pr}[X \geq 4] \geq 1-\left(1-\frac{3}{n}\right)^{n}-$ $3\left(1-\frac{3}{n}\right)^{n-1}-\frac{9}{2}\left(1-\frac{1}{n}\right)\left(1-\frac{3}{n}\right)^{n-2}-\frac{9}{2}\left(1-\frac{1}{n}\right)\left(1-\frac{2}{n}\right)\left(1-\frac{3}{n}\right)^{n-3}$. By Lemma 3 , $\left(1-\frac{3}{n}\right)^{n}$ and $3\left(1-\frac{3}{n}\right)^{n-1}$ are increasing in $n$ and tend to $e^{-3}$. We shall argue that $\frac{9}{2}\left(1-\frac{1}{n}\right)\left(1-\frac{3}{n}\right)^{n-2}+\frac{9}{2}\left(1-\frac{1}{n}\right)\left(1-\frac{2}{n}\right)\left(1-\frac{3}{n}\right)^{n-3}$ is decreasing, hence it is at most $\frac{124416}{235298}$ for $n \geq 7$. Hence $\operatorname{Pr}[X \geq 4] \geq 1-4 e^{-3}-\frac{124416}{235298} \geq 0.2720$. To see that $\frac{9}{2}\left(1-\frac{1}{n}\right)\left(1-\frac{\overline{3}}{n}\right)^{n-2}+\frac{9}{2}\left(1-\frac{1}{n}\right)\left(1-\frac{2}{n}\right)\left(1-\frac{3}{n}\right)^{n-3}$ is decreasing, we rewrite this term as $f(n)=9\left(1-\frac{1}{n}\right)\left(1-\frac{5}{2 n}\right)^{n}\left(1-\frac{3}{n}\right)^{n-3}$. In a similar fashion as in the proof of Lemma 3, we see that $\ln \left(\frac{1}{9} f(n)\right)=-3+\sum_{i=1}^{\infty} \frac{3^{i+1}-\left(\frac{5}{2}\right)^{i}(i+1)-(i+1)}{i(i+1)} n^{-i}$, which is a sum of constant and decreasing function.

For the case $k=n-4$ and $n \geq 8$, we compute $\operatorname{Pr}[X \geq n-3]=\frac{4^{3}}{6}\left(1-\frac{1}{n}\right)(1-$ $\left.\frac{2}{n}\right)\left(1-\frac{4}{n}\right)^{n-3}+8\left(1-\frac{1}{n}\right)\left(1-\frac{4}{n}\right)^{n-2}+4\left(1-\frac{4}{n}\right)^{n-1}+\left(1-\frac{4}{n}\right)^{4}>\frac{4^{3}}{6}\left(1-\frac{1}{n}\right)\left(1-\frac{4}{n}\right)^{n-2}+$ $8\left(1-\frac{1}{n}\right)\left(1-\frac{4}{n}\right)^{n-2}+4\left(1-\frac{4}{n}\right)^{n-1}+\left(1-\frac{4}{n}\right)^{4}$, which is increasing in $n$ by Lemma 3 , Using $n \geq 8$, we conclude $\operatorname{Pr}[X \geq n-3] \geq 0.2903$.

We now note that $g(12,4)=g(12,8)>0.25$ and that $g(11,5)=g(11,6)>$ 0.25 . With the same monotonicity arguments as above, this solves all cases $(n, k)$ which can be written as $(n, k)=\left(n_{0}+i+j, k_{0}+j\right)$ with $i, j \in \mathbb{N} \cup\{0\}$ and $\left(n_{0}, k_{0}\right) \in\{(12,4),(11,5),(11,6),(12,8)\}$. This leaves to check only the cases $(n, k) \in\{(9,4),(10,4),(11,4),(10,5)\}$. These are best computed by hand, e.g., $\operatorname{Pr}\left[\operatorname{Bin}\left(9, \frac{4}{9}\right) \geq 5\right] \geq \operatorname{Pr}\left[\operatorname{Bin}\left(9, \frac{4}{9}\right)=5\right]+\operatorname{Pr}\left[\operatorname{Bin}\left(9, \frac{4}{9}\right)=6\right] \geq 0.2081+0.1110=$ 0.31911

Since GM14] showed a probability of strictly more than $\frac{1}{4}$ (albeit for the weaker event $X \geq E[X]$ ), let us remark that our proof above also shows $\operatorname{Pr}[X>E[X]]>\frac{1}{4}$ for all $n \in \mathbb{N}$ and $\ln \left(\frac{4}{3}\right) / n \leq p<1$ except $(n, p)=\left(2, \frac{1}{2}\right)$, where indeed $\operatorname{Pr}[X>$ $E[X]]=\frac{1}{4}$. The proof above does not exclude $\operatorname{Pr}\left[\operatorname{Bin}\left(n, \frac{k}{n}\right)=k+1\right]=\frac{1}{4}$ only for the case that $n \geq 2$ and $k=n-1$. The success probability shown above is (strictly) increasing in $n$, so the one remaining case is $(n, k)=(2,1)$, where indeed we do not have $\operatorname{Pr}[X>E[X]]>\frac{1}{4}$. By the strict domination result of Lemma 7 , for $p \notin\left\{\frac{1}{n}, \frac{2}{n}, \ldots, \frac{n-1}{n}\right\}$ we always have $\operatorname{Pr}[X>E[X]]>\frac{1}{4}$.

\footnotetext{
${ }^{1}$ More precisely, for $(n, k)=(9,4),(10,4),(11,4),(10,5)$ we have $\operatorname{Pr}\left[\operatorname{Bin}\left(n, \frac{k}{n}\right) \geq k+1\right] \approx$ $0.3655,0.3668,0.3678,0.3769$, respectively, where all values have been rounded down to the nearest multiple of 0.0001 .
} 

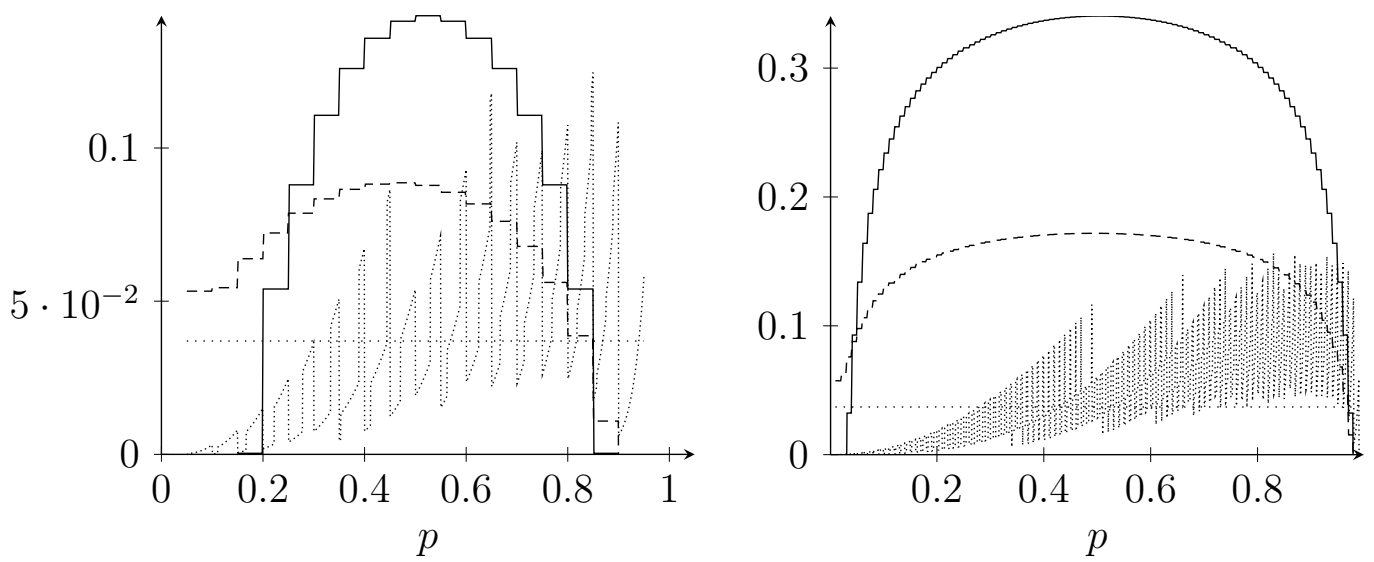

Figure 3: Comparison of the lower bounds for $\operatorname{Pr}[\operatorname{Bin}(n, p)>E[X]+1]$ stemming from Theorem 11 [Pel16] (dotted lines) and our Theorem 12 (a) (solid lines), (b) (dashed lines), and (c) (sparsely dotted lines) for $n=20$ (left) and $n=100$ (right).

\section{Exceeding the Expectation by More Than One}

We end this section with a short proof of the fact that binomial random variables exceed their expectation also by more than one with constant probability (obviously only when $p<1-\frac{1}{n}$ ). For this problem, not much previous work exists. Pelekis [Pel16] shows the following estimate for exceeding the expectation by general amounts.

Theorem 11. Let $n \in \mathbb{N}, 0<p<1$, and $n p<k \leq n-1$. With $\ell:=\left\lfloor\frac{k-n p}{1-p}\right\rfloor$, we have

$$
\operatorname{Pr}[X \geq k] \geq \frac{p^{2 \ell+2}}{2} \frac{\left(\begin{array}{c}
n \\
\ell+1
\end{array}\right)}{\left(\begin{array}{c}
k \\
\ell+1
\end{array}\right)} .
$$

For the case of exceeding the expectation by more than one, we show the following results. As Figure 3 indicates, Pelekis' and our bounds do not compare easily, but ours give the more uniform results.

Theorem 12. Let $n \in \mathbb{N}_{\geq 3}$ and $\frac{1}{n} \leq p<1-\frac{1}{n}$. Let $X \sim \operatorname{Bin}(n, p)$. Let $k=\lfloor n p\rfloor$. Then

(a) $\operatorname{Pr}[X>E[X]+1] \geq 0.5-2 \sqrt{\frac{n}{2 \pi k(n-k)}}$;

(b) $\operatorname{Pr}[X>E[X]+1] \geq 0.25-\sqrt{\frac{n}{2 \pi(k+1)(n-k-1)}}\left(1-\frac{1}{k+1}\right)^{k+1}\left(1+\frac{1}{n-k-1}\right)^{n-k-1}$;

(c) $\operatorname{Pr}[X>E[X]+1] \geq 0.0370$. 
Proof. Let $Y \sim \operatorname{Bin}\left(n, \frac{k}{n}\right)$. By Lemma 7, we have

$$
\operatorname{Pr}[X>E[X]+1]=\operatorname{Pr}[X \geq k+2] \geq \operatorname{Pr}[Y \geq k+2],
$$

so we can assume in the following that $p=\frac{k}{n}$ with $k \in[1 . . n-2]$ and show our claims for $\operatorname{Pr}[X \geq k+2]$.

Similar to the proof of Lemma 8 , we compute

$$
\begin{aligned}
\operatorname{Pr}[X \geq k+2] & =\operatorname{Pr}[X \geq k]-\operatorname{Pr}[X=k]-\operatorname{Pr}[X=k+1] \\
& \geq \operatorname{Pr}[X \geq k]-2 \operatorname{Pr}[X=k] \\
& >\frac{1}{2}-2 \sqrt{\frac{n}{2 \pi k(n-k)}}
\end{aligned}
$$

where the estimate $\operatorname{Pr}[X=k] \geq \operatorname{Pr}[X=k+1]$ used in the penultimate inequality either follows from a simple computation or from the well-known fact that the mode of $\operatorname{Bin}\left(n, \frac{k}{n}\right)$ is $k$.

By using Theorem 10 and Corollary [5, we compute

$$
\begin{aligned}
\operatorname{Pr} & {[X \geq k+2]=\operatorname{Pr}[X>E[X]]-\operatorname{Pr}[X=E[X]+1] } \\
& \geq \frac{1}{4}-\sqrt{\frac{n}{2 \pi(k+1)(n-k-1)}}\left(1-\frac{1}{k+1}\right)^{k+1}\left(1+\frac{1}{n-k-1}\right)^{n-k-1} \\
& :=h(n, k) .
\end{aligned}
$$

To prove the last claim, recall that we have to show

$$
\operatorname{Pr}\left[\operatorname{Bin}\left(n, \frac{k}{n}\right) \geq k+2\right] \geq 0.0370
$$

for all $n \geq 3$ and $k \in[1 . . n-2]$. To this aim, we first exploit in two different ways the lower bound $h(n, k)$.

By rewriting

$$
\sqrt{\frac{n}{n-k-1}}\left(1+\frac{1}{n-k-1}\right)^{n-k-1}=\sqrt{\frac{n}{n-k}}\left(1+\frac{1}{n-k-1}\right)^{n-k-1+0.5}
$$

as product of two terms which are both decreasing in $n$ for $n>k+1$, see Lemma 3 (b), we observe that $h(n, k)$ is increasing for $k$ fixed and $n \geq k+2$ growing. Since $h(n, k)>0.0370$ for $(n, k) \in\{(6,1),(9,2),(9,3),(10,4),(10,5)\}$.2 we have shown (66) for all

$$
(n, k) \in\{(6+i, 1),(9+i, 2),(9+i, 3),(10+i, 4),(10+i, 5) \mid i \geq 0\} .
$$

\footnotetext{
${ }^{2}$ More precisely, we have $h(6,1) \approx 0.0391, h(9,2) \approx 0.0392, h(9,3) \approx 0.0392, h(10,4) \approx$ 0.0442 , and $h(10,5) \approx 0.0394$, where the value given is the true value rounded down to the nearest multiple of 0.0001 .
} 
We now argue that $h(n, n-5)$ is increasing in $n$ for $n \geq 10$. We rewrite the relevant part $\sqrt{\frac{n}{n-4}}\left(1-\frac{1}{n-4}\right)^{n-4}=\left(1+\frac{4}{n-4}\right)^{1 / 2}\left(1-\frac{1}{n-4}\right)\left(1-\frac{1}{n-4}\right)^{(n-4)-1}$. The last factor is known to be decreasing for $n>4$. For the first two factors, we compute $\left(\left(1+\frac{4}{n-4}\right)^{1 / 2}\left(1-\frac{1}{n-4}\right)\right)^{2}=1+\frac{2}{n-4}-\frac{7}{(n-4)^{2}}+\frac{4}{(n-4)^{3}}$, which is decreasing from $n-4 \geq 6$ on. Since $h(10,5)>0.0370$, we have $h(n, k)>0.0370$ for all $(n, k) \in\{(10+i, 5+i) \mid i \geq 0\}$, and via the first monotonicity statement for all

$$
(n, k) \in\{(10+i+j, 5+i) \mid i, j \geq 0\} .
$$

For the remaining cases, we estimate directly the probability $q(n, k):=$ $\operatorname{Pr}\left[\operatorname{Bin}\left(n, \frac{k}{n}\right) \geq k+2\right]$ in (6) $)$. For $k \in\{n-2, n-3, n-4\}$, we simply compute

$$
\begin{aligned}
& q(n, n-2)=\left(1-\frac{2}{n}\right)^{n} \geq 0.0370 \\
& q(n, n-3)=\left(1-\frac{3}{n}\right)^{n}+3\left(1-\frac{3}{n}\right)^{n-1} \geq 0.0507 \\
& q(n, n-4)=\left(1-\frac{4}{n}\right)^{n}+4\left(1-\frac{4}{n}\right)^{n-1}+8\left(1-\frac{1}{n}\right)\left(1-\frac{4}{n}\right)^{n-2} \geq 0.0579,
\end{aligned}
$$

where the last estimates stem from noting that all the terms involved are increasing in $n$ by Lemma (a) and evaluating the expressions for $n=3, n=4$, and $n=5$, respectively. This shows (6) for all

$$
(n, k) \in\{(3+i, 1+i),(4+i, 1+i),(5+i, 1+i) \mid i \geq 0\} .
$$

For the last four cases $(n, k) \in\{(7,2),(8,2),(8,3),(9,4)\}$, equation (마) is easily checked by hand: We have $q(7,2) \approx 0.1082, q(8,2) \approx 0.1138, q(8,3) \approx 0.1374$, and $q(9,4) \approx 0.1573$.

We note that, as in Corollary 9, smaller values of $p$ can be admitted at the price of a smaller probability for the event " $X>E[X]+1$ ". For $p=\frac{\alpha}{n}<\frac{1}{n}$, we have $\operatorname{Pr}[X>E[X]+1]=1-(1-p)^{n}-n p(1-p)^{n-1}=1-\left(1-\frac{\alpha}{n}\right)^{n}-\alpha\left(1-\frac{\alpha}{n}\right)^{n-1} \geq$ $1-\exp (-\alpha)-\alpha \exp \left(-\alpha \frac{n-1}{n}\right)$.

\section{Acknowledgment}

The author would like to thank Philippe Rigollet (Massachusetts Institute of Technology (MIT)) and Carsten Witt (Danish Technical University (DTU)) for useful discussions and pointers to the literature.

\section{References}

$\left[\mathrm{BCN}^{+} 17\right]$ Luca Becchetti, Andrea E. F. Clementi, Emanuele Natale, Francesco Pasquale, Riccardo Silvestri, and Luca Trevisan. Simple dynamics for plurality consensus. Distributed Computing, 30:293-306, 2017. 
[DGWY17] Benjamin Doerr, Christian Gießen, Carsten Witt, and Jing Yang. The $(1+\lambda)$ evolutionary algorithm with self-adjusting mutation rate. In Genetic and Evolutionary Computation Conference, GECCO 2017. ACM, 2017. Full version available at http://arxiv.org/abs/1704.02191.

[Fel71] William Feller. An Introduction to Probability Theory and Its Applications, volume II. John Wiley \& Sons Inc., New York, second edition, 1971.

[GM14] Spencer Greenberg and Mehryar Mohri. Tight lower bound on the probability of a binomial exceeding its expectation. Statistics and Probability Letters, 86:91-98, 2014.

[KKK16] Matti Karppa, Petteri Kaski, and Jukka Kohonen. A faster subquadratic algorithm for finding outlier correlations. In Proceedings of the Twenty-Seventh Annual ACM-SIAM Symposium on Discrete Algorithms, SODA 2016, pages 1288-1305. SIAM, 2016.

[MM17] Michael Mitzenmacher and Tom Morgan. Reconciling graphs and sets of sets. CoRR, abs/1707.05867, 2017.

[Neu66] P. Neumann. Über den Median der Binomial- and Poissonverteilung. Wissenschaftliche Zeitschrift der Technischen Universität Dresden, 19:29-33, 1966.

[Pel16] Christos Pelekis. A lower bound on binomial tails: an approach via tail conditional expectations. ArXiv e-prints, 1609.06651, 2016.

[PR16] Christos Pelekis and Jan Ramon. A lower bound on the probability that a binomial random variable is exceeding its mean. Statistics and Probability Letters, 119:305-309, 2016.

[Rob55] Herbert Robbins. A remark on Stirling's formula. American Mathematical Monthly, 62:26-29, 1955.

[RT11] Philippe Rigollet and Xin Tong. Neyman-Pearson classification, convexity and stochastic constraints. Journal of Machine Learning Research, 12:2831-2855, 2011.

[Slu77] Eric V. Slud. Distribution inequalities for the binomial law. Annals of Probability, 5:404-412, 1977. 